This information is current as of April 25, 2023. 


\title{
ORIGINAL RESEARCH \\ Unilateral Hemispheric Proliferation of Ivy Sign on Fluid-Attenuated Inversion Recovery Images in Moyamoya Disease Correlates Highly with Ipsilateral Hemispheric Decrease of Cerebrovascular Reserve
}

M. Kawashima

T. Noguchi

Y. Takase

T. Ootsuka

N. Kido

T. Matsushima

\begin{abstract}
BACKGROUND AND PURPOSE: An ivy sign is considered to represent diffuse leptomeningeal collaterals found on fluid-attenuated inversion recovery (FLAIR) images of patients with Moyamoya disease. We evaluated the correlation between unilateral ivy proliferation in a hemisphere and cerebrovascular hemodynamic status to learn the clinical significance of the ivy sign.
\end{abstract}

\begin{abstract}
MATERIALS AND METHODS: A total of 35 patients with Moyamoya disease were included. Correlation between ivy dominance on FLAIR images and hemodynamic status with use of iodine $123 \mathrm{~N}$-isopropylp-iodoamphetamine ( ${ }^{123}$ /-IMP) single-photon emission CT (SPECT) was evaluated.
\end{abstract}

RESULTS: Distributional differences of ivy signs between both hemispheres were observed in 22
$(64.7 \%)$ of 34 patients with a positive ivy sign, all of whom showed decreased vascular reserve/
reactivity in the ivy-dominant hemisphere (IDH). The proportion of the stage II (misery perfusion) area
to IDH was higher than that in the ivy less-dominant hemisphere (ILDH) in the quantitative analysis.
The mean vascular reserve was lower in IDH than ILDH. There were 15 of 22 patients who had bypass
surgery on IDH because of transient ischemic attack from ischemia of IDH. Patients with symmetric
ivy distributions showed a variety of hemodynamic status. MR angiography (MRA) stage of IDH
$(2.95 \pm 0.39)$ was higher compared with ILDH $(2.60 \pm 0.50 ; P<.05)$. Regional arteriocapillary
circulation time ratio in IDH was longer compared with ILDH $(P<.05)$. Ivy proliferation decreased in
$10(55.6 \%)$ of 18 patients who underwent bypass surgery during the follow-up period.

CONCLUSIONS: Unilateral hemispheric ivy proliferation correlated highly with the existence of an ipsilateral decreased vascular reserve associated with the development of leptomeningeal collaterals in patients with Moyamoya disease.

vy sign refers to a diffuse leptomeningeal enhancement found on postcontrast MR images of patients with Moyamoya disease in childhood. ${ }^{1}$ The ivy sign is also seen in a child with Moyamoya disease on fluid-attenuated inversion-recovery (FLAIR) images. ${ }^{2}$ The mechanism of this enhancement is considered to represent a retrograde slow flow of engorged pial vasculature toward an ischemic area via leptomeningeal collaterals. ${ }^{1,2}$ The clinical significance of the ivy sign, however, remains unclear. The purpose of our study was to evaluate the correlation between ivy proliferation in the hemispheres and cerebrovascular hemodynamic status, focusing especially on the vascular reserve measured by ${ }^{123}$ I-iodoamphetamine (IMP) single-photon emission CT (SPECT) with acetazolamide (ACZ) challenge.

\section{Materials and Methods}

\section{Patients}

A total of 35 patients who presented with transient ischemic attack (TIA; $n=23)$, headache $(n=6)$, no symptoms $(n=5)$, and incontinence $(n=1)$ were diagnosed as having Moyamoya disease during 2

Received March 2, 2009; accepted after revision April 20.

From the Departments of Neurosurgery (M.K., Y.T., T.M.) and Radiology (T.N., T.O., N.K.), Faculty of Medicine, Saga University, Saga, Japan.

Please address correspondence to Masatou Kawashima, MD, PhD, Department of Neurosurgery, Faculty of Medicine, Saga University, 5-1-1 Nabeshima, Saga 849-8501 Japan; e-mail: MasatouAzu@aol.com

DOI 10.3174/ajnr.A1679 years at our institution. A total of 25 patients were female (average age, 29.1 years; age range, 7-49 years), and 10 were male (average age, 18.3 years; age range, from 7-54 years). Medical treatment for patients was determined after the evaluation of clinical conditions and symptoms, MR imaging and MR angiography (MRA), conventional angiography (if available), and cerebrovascular reserve by use of SPECT with ACZ challenge. For symptomatic patients, indirect (encephaloduroarteriosynangiosis) or direct (superficial temporal artery-middle cerebral artery anastomosis) bypass was planned for the hemisphere without a major infarction, where the cerebrovascular reserve was decreased.

\section{Imaging Examinations}

MR Imaging and MRA. The routine MR imaging protocol for Moyamoya disease includes FLAIR and MRA images. Moyamoya disease was diagnosed in all patients with use of MRA. Postoperative MR imaging follow-up (average, 10.1 months) was performed for all surgically treated patients. MR imaging was performed with a 3T MR unit (Trio; Siemens Medical Systems, Erlangen, Germany). FLAIR imaging was obtained with a fast FLAIR sequence with a TR of 9000 $\mathrm{ms}$, an effective TE of $86 \mathrm{~ms}$, an inversion time of $2500 \mathrm{~ms}$, a bandwidth of $241 \mathrm{~Hz} /$ pixel, and a flip angle of $150^{\circ}$. Image thickness, gap, and matrix for all sequences were $6.0 \mathrm{~mm}, 1.2 \mathrm{~mm}$, and $384 \times$ 372 pixels, respectively. A 3D time-of-flight MRA was obtained with a TR of $22 \mathrm{~ms}$, an TE of $3.1 \mathrm{~ms}$, a bandwidth of $250 \mathrm{~Hz} /$ pixels, and a flip angle of $18^{\circ}$. Voxel size was $0.52 \times 0.52 \times 0.8 \mathrm{~mm}$, and acquisition matrix was $384 \times 320$.

An ivy sign on a FLAIR image was defined as a continuous linear 

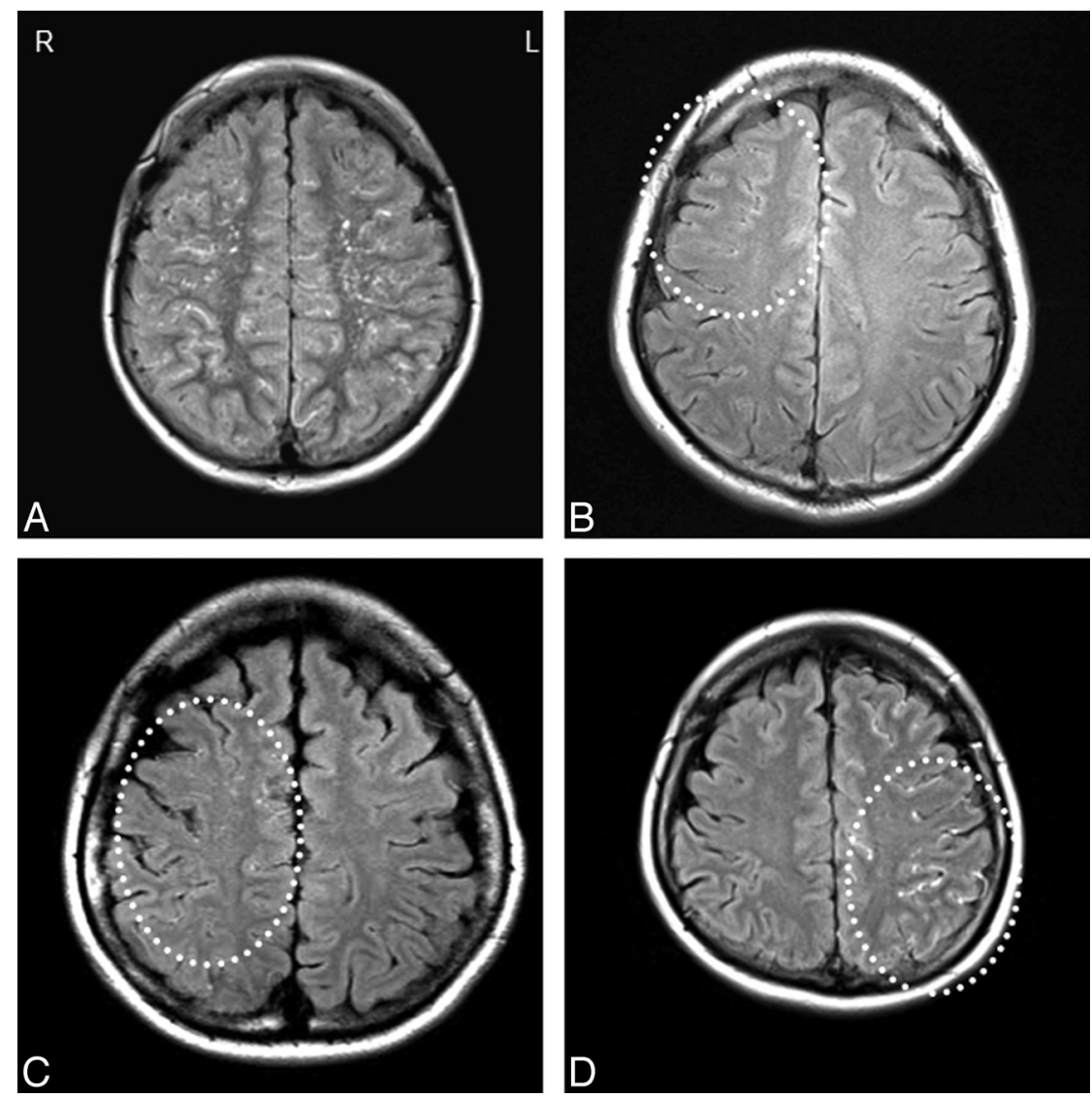

Fig 1. The proliferative differences of ivy signs on FLAIR images between both hemispheres in each patient was rated as minimal, moderate, or marked. $A$, Symmetric ivy distribution. We defined the hemispheres as ivy symmetric when a symmetric ivy distribution was observed in both hemispheres. $B$, Minimal ivy proliferation along the cortical sulci dominantly in the right frontal lobe (dotted circle). C, moderate ivy dominance in the right hemisphere (dotted circle). D, Marked ivy distribution in the left hemisphere (dotted circle).

or punctate leptomeningeal high signal intensity along the cortical sulci and subarachnoid space. Two neuroradiologists retrospectively reviewed MR images for the ivy sign on FLAIR images and MRA images. We defined the hemispheres as ivy unsymmetric when the proliferative difference of ivy signs on FLAIR images was observed between both hemispheres in each patient. This applies to the patient with no ivy proliferation in the contralateral hemisphere. We defined the hemispheres as ivy symmetric when a symmetric ivy distribution was observed in both hemispheres (Fig 1A). We also defined the ivydominant hemisphere as IDH and ivy less-dominant hemisphere as ILDH in the patients with an unsymmetric ivy sign. The relative proliferative differences of ivy signs on FLAIR images between hemispheres in each patient was rated as "minimal" (Fig $1 B$ ), "moderate" (Fig 1C), or "marked" (Fig 1D). Minimal proliferation indicating subtle and equivocal high signal intensity along the cortical sulci was seen dominantly in either hemisphere, whereas marked proliferation indicating obvious linear and punctate high signal intensity was encountered dominantly in either hemisphere.

The correlation between hemispheric ivy proliferation and MRA images was studied according to the MRA grading system. ${ }^{3,4}$ MRA scores of IDH and ILDH were compared. MRA scores were based on the severity of occlusive changes of the internal carotid artery, the horizontal portion of the middle cerebral artery (MCA), the anterior and the posterior cerebral arteries (ACAs and PCAs), and signals of the distal branches of these arteries. Total points ranged from 0 (normal) to 10 (most severe). Four stages on the basis of this novel MRA score correlated well with Suzuki stages, with high sensitivity and
Table 1: MRA score in Moyamoya patient*

\begin{tabular}{lll}
\hline ICA & Normal & 0 \\
& Stenosis at supraclinoid portion & 1 \\
& Loss of continuous signal intensity at & 2 \\
& $\quad$ supraclinoid portion & \\
MCA & Invisible & 3 \\
& Normal & 0 \\
& Stenosis at M1 & 1 \\
& Loss of continuous signal intensity at M1 & 2 \\
ACA & Invisible & 3 \\
& Distal to A2 is normal & 0 \\
& Stenosis of ACA distal to A2 & 1 \\
PCA & Invisible & 2 \\
& Distal to P2 is normal & 1 \\
& Stenosis of PCA distal to P2 & 2 \\
& Invisible &
\end{tabular}

Note:-MRA indicates MR angiography; ICA, internal carotid artery; MCA, middle cerebral artery; ACA, anterior cerebral artery; PCA, posterior cerebral artery * MRA score 0-1, MRA grade I; MRA score 2-4, MRA grade II; MRA score 5-7, MRA grade III; MRA score 8-10, MRA grade IV.

specificity (Table 1). ${ }^{3}$ The correlation between ivy proliferation and MRA stage was examined in each patient.

Analysis of circulation time in angiographic examination. For 16 patients, we performed cerebral angiography using the Seldinger method and a digital subtraction radiographic angiographic system (Axion Artis; Siemens). The circulation time was determined by a consensus of 2 authors (M.K., T.N.) observing a serial display system. 
We estimated circulation time by measuring the interval between the time at which the contrast medium filled the siphon portion of the internal carotid artery and the time at which the capillary stain reached its maximal attenuation. ${ }^{5}$ This interval was defined as the regional arteriocapillary circulation time (rACCT) and was evaluated in the bilateral MCA regions of each patient. The ratio of rACCT in IDH to ILDH in patients with an unsymmetric ivy sign was compared with the ratio of the rACCT in the right to left hemispheres in patients with a symmetric ivy sign.

Technique of SPECT. SPECT scanner MULTISPECT3 (Siemens) and image processor ICONP (Siemens) were used. The collimator used was a low-energy, ultra-high resolution, fan-beam collimator (225 cpm/ $\mu \mathrm{Ci})$. Conditions for acquisition were as follows: 20minute acquisition (40 s/90 steps, $3^{\circ}$ step, step-and-shoot method), matrix of $128 \times 128(2.89-\mathrm{mm} /$ pixel $)$, and radius of rotation of 13.9 $\mathrm{cm}$. A filtered backprojection method with a Butterworth filter was used to reconstruct images from 20 -minute data. As a $3 \mathrm{D}$ postfilter, a low-pass filter (cutoff value, $0.3 \mathrm{cycle} / \mathrm{cm}$; order, 5.0) was used. Two SPECT images were summated to obtain a section thickness of 5.78 $\mathrm{mm}$.

All patients underwent 2 SPECT sessions: a basal study and an ACZ-challenged study. The patients were permitted to rest in a quiet, dimly lit room and were intravenously (IV) injected with ${ }^{123} \mathrm{I}-\mathrm{N}$ isopropyl-p-iodoamphetamine $\left({ }^{123} \mathrm{I}\right.$-IMP). We obtained basal images first after injecting the radiotracer. Consecutively, on the same day for semiquantitative SPECT or several days later (3-5 days) for quantitative SPECT, the patients were placed in the same environment as that of the first session and then were IV injected with $1 \mathrm{~g}$ of ACZ (Diamox; Lederle Lab Division, Pearl River, NY) during a period of 1 minute. Fifteen minutes later, the patients were injected with ${ }^{123}$ I-IMP, and the ACZ-challenged images were obtained in the same manner as in the basal study.

\section{Basal/ACZ Stress Brain Perfusion SPECT}

We used 2 different SPECT measurements: quantitative $(n=13)$ and semiquantitative $(n=21)$ for the estimation of vascular reserve/reactivity in patients with Moyamoya disease.

Quantitative Estimation of Vascular Reserve and Stage II Area. In 13 patients including 8 with unsymmetric ivy signs and 5 with ivy symmetric signs, both regional cerebral blood flow (rCBF) and vascular reserve were quantified by autoradiographic processing a single SPECT scan with a 1-point arterial blood sample (IMP-ARG method). ${ }^{6}$ At 10 minutes after beginning ${ }^{123}$ I-IMP (166.5 MBq) infusion, 1 arterial blood sample was taken to calibrate the previously determined standard input function, and its whole-blood radioactivity concentration was counted with a well counter that had been crosscalibrated with the SPECT scanner. A single SPECT scan was performed at a midscan time of 10 minutes after arterial blood sampling. Basal images were obtained 35 minutes after injecting the radiotracer. In a 2-compartment analysis of ${ }^{123} \mathrm{I}$-IMP, distribution volume, the ratio of influx constant and efflux constant can be set to a constant value $(41.2 \mathrm{~mL} / \mathrm{mL})$. rCBF maps were calculated pixel by pixel from single SPECT data, and standard input function was calibrated with a 1-point arterial blood sample. ${ }^{6}$ We also obtained Diamox-activated rCBF maps using the same methods as in the basal study several days later (3-5 days). To objectively classify the severity of hemodynamic ischemia according to the Japanese Extracranial-Intracranial Bypass Trial (JET) study protocol of patients with intracranial occlusive disease, ${ }^{7}$ we performed a quantitative analysis using 3D stereotactic surface projections analytical software (3D-SSP; Nihon Mediphysics,
Tokyo, Japan) and stereotactic extraction estimation display software (SEE-JET; Nihon Mediphysics). ${ }^{8}$ This method provides quantitative information and 3D displays of the CBF at rest and after Diamox challenge, vascular reserve, and severity of hemodynamic brain ischemia. Regional vascular reserve was defined as the ratio of the difference between Diamox-activated $\mathrm{rCBF}$ and resting rCBF to resting rCBF [(Diamox-activated rCBF/resting rCBF-1) $\times 100(\%)]$. Resting rCBF, Diamox-activated rCBF, and regional vascular reserve of bilateral ACA, MCA, and PCA distributions were evaluated at the hemispheres. The severity of hemodynamic brain ischemia was classified into the 3 stages: stage 0 (resting CBF $>15 \mathrm{~mL} / 100 \mathrm{~g} / \mathrm{min}$ and vascular reserve $>30 \%)$, stage $\mathrm{I}[34 \mathrm{~mL} / 100 \mathrm{~g} / \mathrm{min}(80 \%$ of normal $\mathrm{CBF})>$ resting $\mathrm{CBF}>15 \mathrm{~mL} / 100 \mathrm{~g} / \mathrm{min}$ and $30 \%>$ vascular reserve $>10 \%$ ], and stage $I I(34 \mathrm{~mL} / 100 \mathrm{~g} / \mathrm{min}>$ resting $\mathrm{CBF}>15 \mathrm{~mL} / 100 \mathrm{~g} / \mathrm{min}$ and $10 \%>$ vascular reserve $>-30 \%){ }^{7,8}$ Finally, the proportion of stage II area to hemisphere (ACA and MCA territories) and mean vascular reserves in hemisphere were compared for IDH and ILDH in patients with an unsymmetric ivy sign and right and left hemispheres in patients with a symmetric ivy sign.

Semiquantitative Estimation of Vascular Reserve. In 21 patients including 14 with ivy unsymmetric sign and 7 with ivy symmetric sign, the 1-day method for IMP SPECT studies at rest and after ACZ challenge was performed. ${ }^{9}$ Two doses of $111 \mathrm{MBq}{ }^{123} \mathrm{I}$-IMP were IV injected for 1 minute, one at the beginning and the other at $30 \mathrm{~min}$ utes after the first IMP injection. ACZ ( $1 \mathrm{~g})$ was slowly IV injected for 1 minute, starting 15 minutes before the end of the initial scan. Among the sections obtained in the basal SPECT study, 1 tomographic plane located 50 to $75 \mathrm{~mm}$ above and parallel to the orbitomeatal line, and showing the most prominent contrast in the ACA and MCA territories, was selected for each patient, and the regions of interest (ROIs) were placed directly on each SPECT image. ROIs were placed in the region where infarction was not present on MR imaging. On each SPECT image, mirror-image ROIs were also placed in the corresponding region on the contralateral side. Identical ROIs were analyzed in each subject for SPECT studies. The ratio of the quantitative radioactivity count obtained from the ROI on the IDH of the MCA territories to that obtained from the ROI on the ILDH was calculated by asymmetry index (AI: mean count on the IDH/mean count on the ILDH) in patients with an ivy unsymmetric sign. ${ }^{5}$ If the dominancy of the ivy side was not determined in a patient, the AI was calculated as the quantitative radioactivity count obtained from the ROIs on the right side of the ACA and MCA territories to that obtained from the left side. For the ACZ-challenged SPECT study, a section was selected at the same level as that selected in the basal study, and the AI was calculated with use of the same methods as in the basal study. The proportion of ACZ-challenged AI to basal AI was then defined as the regional reactivity index (rRI). Finally, mean RIs of the ACA and MCA territories were compared between the patients with an unsymmetric ivy sign and patients with a symmetric ivy sign.

\section{Statistical Analysis}

The statistical analysis was performed with the Student $t$ test for correlations between patients with an ivy symmetric sign and patients with an ivy unsymmetric sign. The difference was considered to be significant if the $P$ value was less than $5 \%(P<.05)$.

\section{Results}

Relationships between ivy sign on MR imaging and patients' clinical symptoms related to TIA (body affected, no hemisphere involved), operation performed, and postoperative 


\begin{tabular}{lcccc}
\hline \multicolumn{5}{l}{ Table 2: Ivy sign and clinical characteristics of $\mathbf{3 4}$ patients } \\
\hline Ivy Dominance & Patients $(n)$ & ${ }^{*}$ TIA (Right/Left) & Bypass Surgery (Right/Left) & Postoperative Ivy Decrease \\
\hline Left side & 12 & $7(58.3 \%) / 0$ & $0 / 8(66.6 \%)$ & $5 / 8(62.5 \%)$ \\
Minimal & 4 & $2 / 0$ & $0 / 1$ & $0 / 5$ \\
Moderate & 5 & $3 / 0$ & $0 / 2$ & $7(70 \%) / 0$ \\
Marked & 3 & $2 / 0$ & $2 / 0$ & $4 / 7(66.7 \%)$ \\
Right side & 10 & $0 / 9(90 \%)$ & $5 / 0$ & 0 \\
Minimal & 2 & $0 / 2$ & $0 / 6$ & $1 / 3(33.3 \%)$ \\
Moderate & 7 & $0 / 1$ & $1 / 1 / 1(25 \%)($ right/left/bilateral) \\
Marked & 1 & $2 / 3 / 1(50 \%)($ right/left/bilateral) &
\end{tabular}

Note:-TIA indicates transient ischemic attack.

* TIA refers to symptomatic body side.
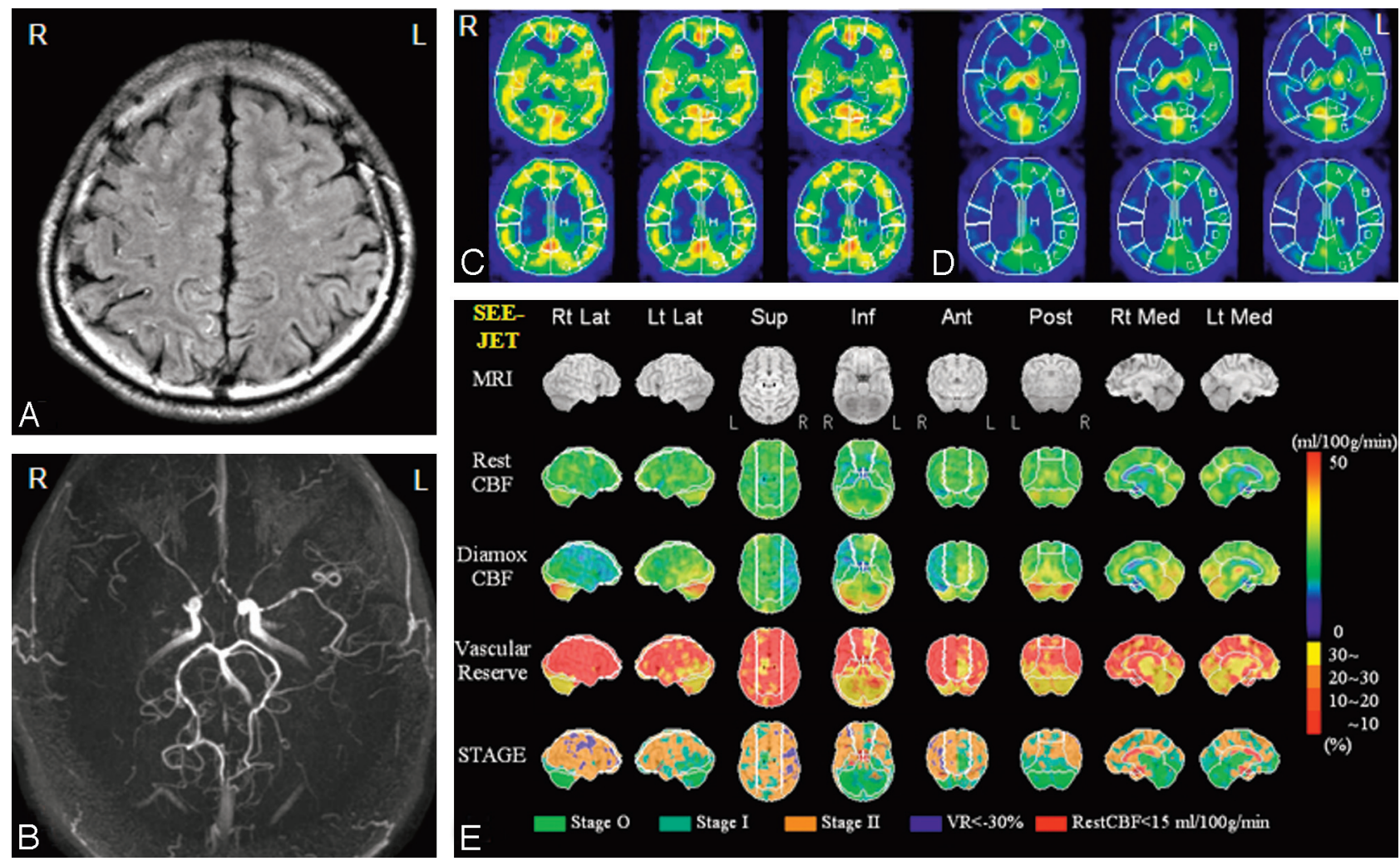

Fig 2. Case of a 28-year-old man with unsymmetric ivy sign and decreased vascular reserve defined by quantitative SPECT analysis. He had sustained a left TIA. $A$, FLAIR image showed marked ivy sign in the right hemisphere. $B$, MRA stages in right and left hemispheres were III and II, respectively. $C$, Basal brain perfusion SPECT showed mildly decreased CBF in the right hemisphere. D, ACZ stress brain perfusion SPECT showed remarkably decreased CBF in the right hemisphere. E, Quantitative analysis of basal/ACZ stress brain perfusion SPECT. Lower 4 columns show 3D-SSP format view sets of rest CBF, Diamox CBF, vascular reserve, and staging by JFT study from top. Vascular reserve was impaired in most of the right ACA and MCA and in part of the left ACA and MCA territories. The proportions of the stage II area in the right and left hemispheres were $64.9 \%$ and $57.9 \%$, respectively. Vascular reserve means in the right and left hemispheres were -9.63 and 2.80 , respectively. Vascular reserve less than $-30 \%$ areas were seen scattered in the right hemisphere. The patient is free from symptoms after cerebral revascularization on the right side.

MR imaging findings are summarized in Table 2. Ivy signs on FLAIR images were observed in 34 (97.1\%) of 35 patients. The proliferation difference of ivy signs between hemispheres in each patient was observed for $22(64.7 \%)$ of 34 patients with positive ivy sign: 12 with left-sided dominance and 10 with right-sided dominance. Four minimal, 5 moderate, and 3 marked hemispheric differences in ivy sign were seen in 12 patients with left-sided dominance. Two minimal, 7 moderate, and 1 marked hemispheric ivy differences were seen in 10 patients with right-sided dominance. Twelve patients showed symmetric ivy proliferation in both hemispheres. Seven (58.3\%) showed right TIA in 12 left-sided ivy-dominant patients, whereas 9 (90\%) showed left TIA in 10 right-sided ivydominant patients. Eight (66.6\%) of 12 left-sided ivy-domi- nant patients had bypass surgery ( 7 indirect bypasses and 1 direct bypass) in the left side, whereas 7 (70\%) of 10 rightsided ivy-dominant patients had bypass surgery (6 indirect bypasses and 1 direct bypass) in the right side. Three (25\%) of 12 patients who showed symmetric ivy proliferation in both hemispheres had bypass surgery in the left, right, and both sides, respectively.

All patients with ivy unsymmetric (Figs $2 A,-B$ ), whether left-side or right-side dominant, showed decreased $\mathrm{rCBF}$ in the IDH compared with ILDH on SPECT with activated clotting time (ACT) challenge. Quantitative SPECT analysis showed an increased proportion of stage II (misery perfusion) area and decreased vascular reserve in the IDH compared with ILDH in all patients with unsymmetric ivy distributions (Figs 

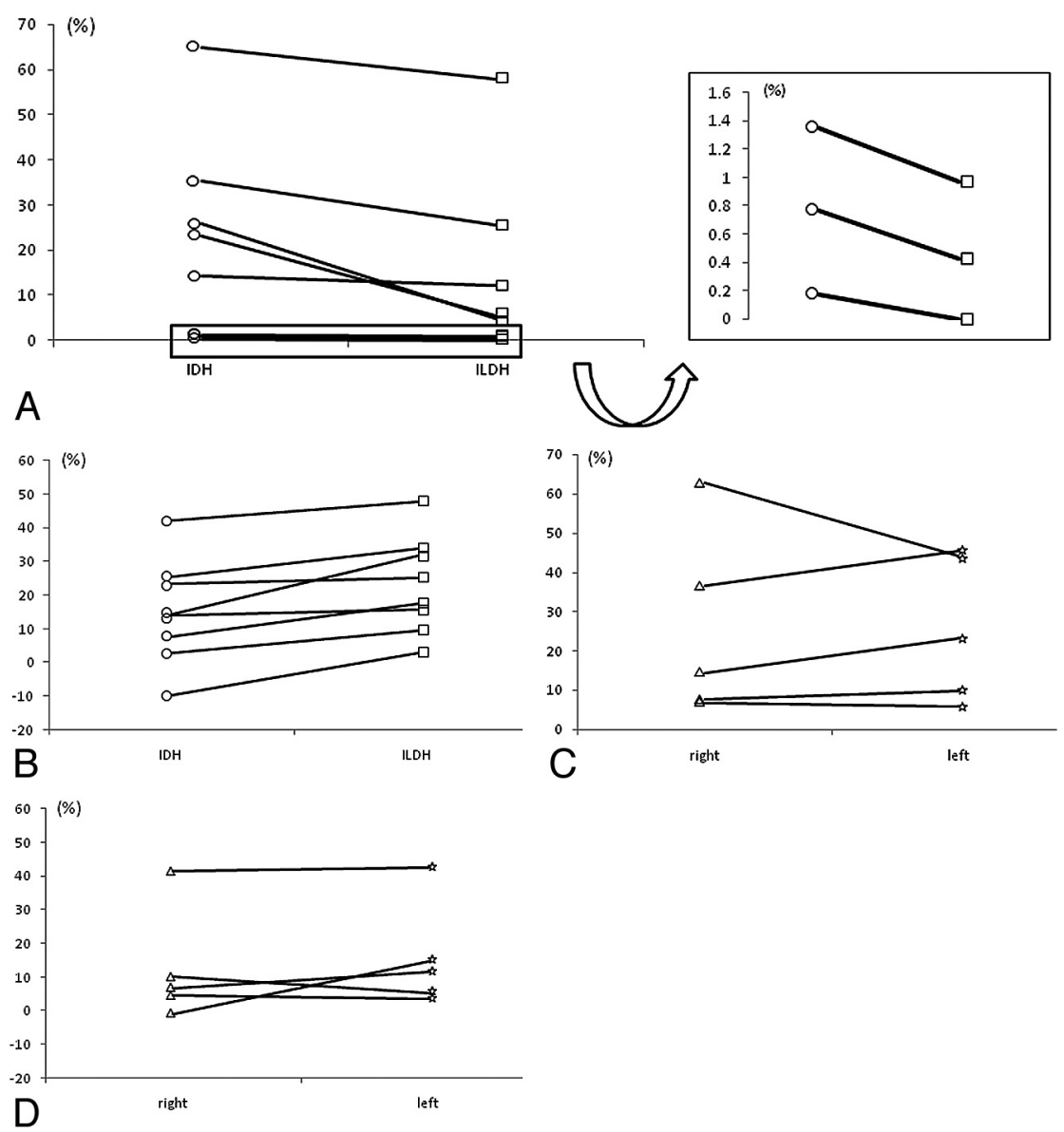

Fig 3. Quantitative estimation of SPECT in 13 patients with ivy unsymmetric and ivy symmetric distributions. IDH indicates ivy dominant hemisphere; ILDH, ivy less dominant hemisphere $A, B$, Proportion of stage II area and mean vascular reserve in IDH and ILDH in patients with an unsymmetric ivy sign $(n=8)$. $A$, Proportion of stage II area in IDH and ILDH. Mean proportions of stage II area are $20.71 \% \pm 22.14 \%$ in IDH and $13.43 \% \pm 19.91 \%$ in ILDH. B, Mean vascular reserve in IDH and ILDH. Mean vascular reserves are $15.06 \% \pm 15.69 \%$ in IDH and $23.00 \%$ $\pm 14.59 \%$ in ILDH. C,D Proportion of stage II area and mean vascular reserve in both hemispheres in patients with symmetric ivy sign ( $n=5)$. $C$, Proportion of stage II area in both hemispheres. Mean proportions of stage II area are $25.64 \% \pm 24.00 \%$ in the right hemisphere and $25.64 \% \pm 18.55 \%$ in the left hemisphere. $D$, Mean vascular reserve in both hemispheres. Mean vascular reserves are $12.38 \% \pm 16.69 \%$ in the right hemisphere and $15.49 \% \pm 15.77 \%$ in the left hemisphere.

Table 3: Semiquantitative estimation of vascular reactivity by SPECT in 21 patients with ivy unsymmetric and ivy symmetric proliferation

\section{RI Mean}

Ivy unsymmetric $(n=14) \quad 0.80 \pm 0.10^{*}$ (Ivy dominant/ivy less dominant) Ivy symmetric $(n=7) \quad 1.05 \pm 0.16$ (right/left)

Note:- $\mathrm{Rl}$ indicates (vascular) reactivity index; SPECT, single-photon emission CT. ${ }^{*} P<.05$

$2 C, E)$. The proportion of the stage II area of the hemisphere and the mean vascular reserve of the IDH were $20.71 \% \pm$ $22.14 \%$ and $15.06 \% \pm 15.69 \%$, whereas those in the ILDH were $13.43 \% \pm 19.91 \%$ and $23.00 \% \pm 14.59 \%$ in the quantitative analysis (Fig $3 A,-B$ ). Patients with symmetric ivy distributions in the hemispheres on MR imaging showed no hemispheric differences associated with stage II area and mean vascular reserve (Fig 3C, $-D$ ). Semiquantitative SPECT analysis showed mean RI in patients with unsymmetric ivy signs $(n=14)$ to be $0.80 \pm 0.10$, whereas mean RI in patients with symmetric ivy signs $(n=7)$ was $1.05 \pm 0.16(P<.05)$ (Table 3, Figs $4 A,-C$ ).

The correlations between ivy proliferation and MRA stage and angiographic findings are summarized in Table 4. Averages of MRA stage in IDH and ILDH were $2.95 \pm 0.39$ and
$2.60 \pm 0.50$, respectively $(P<.05)$. Averages of MRA stage in the left and right hemispheres of patients with a symmetric ivy sign were $2.78 \pm 0.39$ and $2.88 \pm 0.33$, respectively $(P=.39)$. Regional ACCT ratio in IDH and ILDH were $2.10 \pm 0.24$ and $1.10 \pm 0.23$, respectively $(P<.05)$ (Figs $4 D,-E)$.

The ivy proliferation decreased or diminished in 10 (55.6\%) of 18 patients who underwent bypass surgery during the follow-up period (average, 10.1 months) (Table 1, Fig 5).

\section{Discussion}

In our study, we demonstrated that unilateral hemispheric proliferation of ivy signs on FLAIR images in Moyamoya disease correlates well with ipsilateral hemispheric decrease of cerebrovascular reserve defined on ACZ stress brain perfusion SPECT. Although it was speculated that the ivy sign represents a retrograde slow flow of engorged pial vasculature via the leptomeningeal collaterals, ${ }^{1,2,10}$ the distribution difference of ivy signs between hemispheres in a patient with Moyamoya disease has not been considered to have clinical significance. Knowing that a hemisphere showing a dominant ivy sign has a smaller cerebrovascular reserve than the other hemisphere in a patient with Moyamoya disease is quite valuable because the result for regional cerebrovascular reserve measured by a 

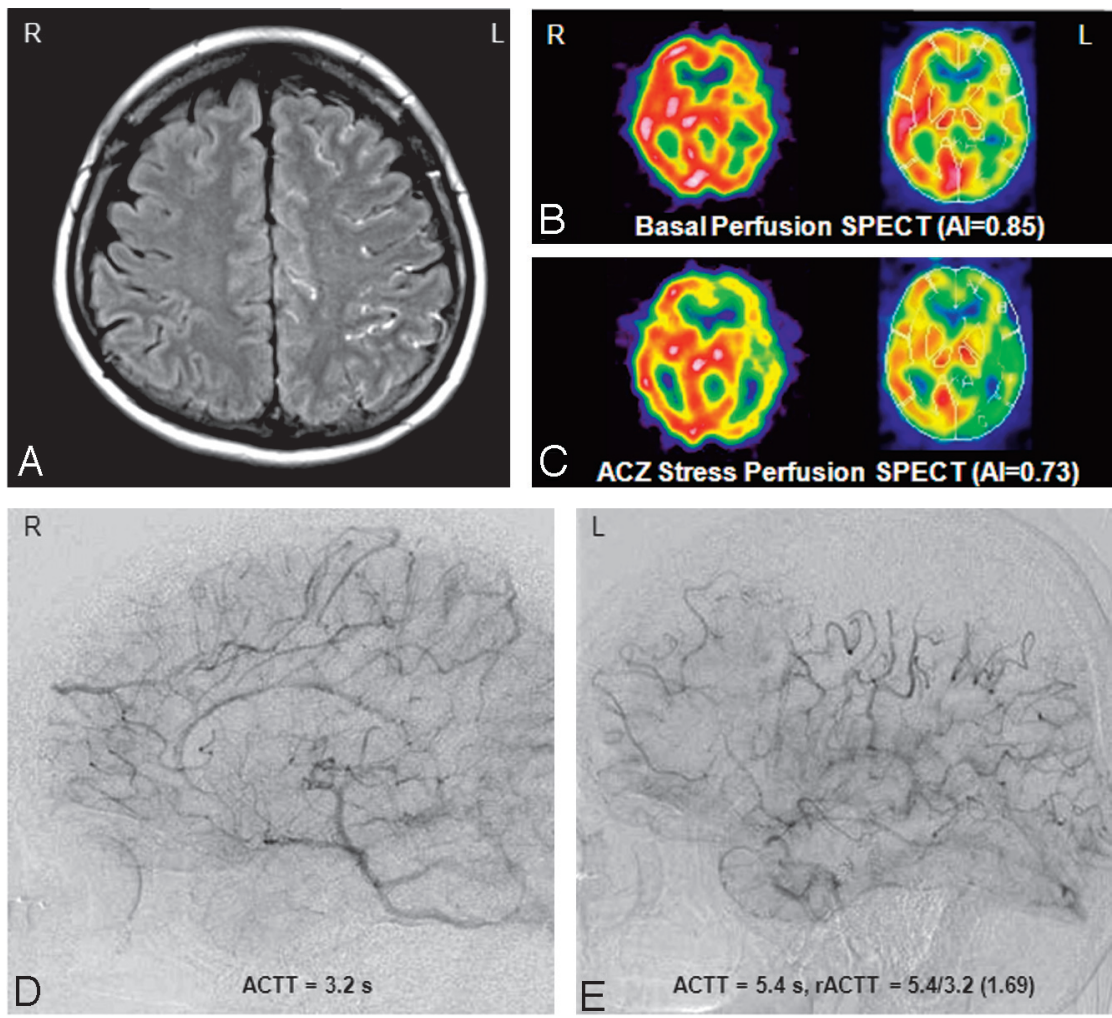

Fig 4. A female patient with unsymmetric ivy sign and decreased RI defined by semiquantitative SPECT analysis. She had sustained a right TIA. A, FLAIR image showed marked ivy sign in the left hemisphere. $B$, Basal brain perfusion SPECT showed decreased CBF in the left hemisphere with an Al of 0.85. $C$, ACZ stress brain perfusion SPECT showed decreased CBF in the left hemisphere with an Al of 0.73. Semiquantitative analysis of basal/ACZ stress brain perfusion SPECT revealed that rRI decreased in the ivy-dominant hemisphere (RI mean, 0.86 ). $D$, Right carotid angiography showed normal filling of vessels in the ACA and MCA regions. E, Left carotid angiography showed delayed filling of vessels in the ACA and MCA regions. A retrograde slow flow of pial vasculature via leptomeningeal collaterals was observed. Regional ACCT in the right and left hemispheres were 3.2 and 5.4 s, respectively (rACCT ratio, 1.69).

\begin{tabular}{llcc}
\hline \multicolumn{4}{l}{ Table 4: Correlation between ivy proliferation and MRA stage and angiographic findings } \\
\hline & MRA Stage (IDH) & MRA Stage (ILDH) & rACCT Ratio \\
\hline Ivy unsymmetric $(n=22)$ & $2.95 \pm 0.39^{*}$ & $2.60 \pm 0.50$ & $2.10 \pm 0.24^{*}(n=9)$ \\
Ivy symmetric $(n=12)$ & $2.78 \pm 0.39$ (left side) & $2.88 \pm 0.33$ (right side) & $1.10 \pm 0.23(n=7)$ \\
\hline
\end{tabular}

Note:- IDH indicates ivy-dominant hemisphere; ILDH, ivy less-dominant hemisphere; rACCT, regional arteriocapillary circulation time.

${ }^{*} P<.05$ (comparison between IDH and ILDH in ivy unsymmetric or right and left in patients with symmetric ivy sign on MRA and comparison between patients with ivy unsymmetric sign and those with ivy symmetric sign of rACTT ratio on angiography).

quantitative examination can be inferred from the existence of ivy dominance on MR imaging. Cerebral revascularization surgery in patients with Moyamoya disease is performed according to their clinical symptoms and image analyses including MR imaging (presence of major infarction or not), MRA or conventional angiography, and cerebral hemodynamic status. Among image analyses, the evaluation of cerebral hemodynamic status is one of the most important factors to consider when deciding surgical indication for cerebral revascularization.

Good candidates for revascularization surgery are those whose cerebral cortex is under misery perfusion, showing an increase of blood flow by SPECT with ACT challenge of less than 0 (steal phenomenon). ${ }^{11,12}$ Positron-emission tomography (PET), xenon-enhanced CT, and blood oxygen level-dependent (BOLD) functional MR imaging as well as SPECT, are used to evaluate cerebral hemodynamic status. ${ }^{13,14}$ BOLD functional MR imaging is a simple, noninvasive method to estimate perfusion and cerebrovascular reserve. ${ }^{14}$ SPECT with ACT challenge, however, is thought to be one of the most convenient and reliable modalities to obtain cerebrovascular reserve and misery perfusion area on the basis of our experience. SPECT examinations were performed not only quantitatively but also semiquantitatively according to the condition of a patient in our institution. It was preferred that children and patients living a long distance from the hospital receive the 1-day method (semiquantitative SPECT analysis). In our study, 22 patients showed an unsymmetric ivy sign intensity on FLAIR images in both hemispheres, all of which had more stage II (misery perfusion) areas in IDH than ILDH, or showed lower vascular reactivity in the IDH than ILDH. Furthermore, the measured mean vascular reserve was lower in the IDH than ILDH in 8 patients receiving quantitative SPECT analyses.

Of 22 patients with an unsymmetric ivy sign, 15 underwent surgical procedures according to their clinical symptoms, angiographic stage, and cerebral perfusion reserve. It is interesting to note that patients with unsymmetric ivy distributions tended to have cerebral revascularization procedures. Retrospective analysis revealed that 13 of 15 surgically treated patients had TIAs (body affected) because of an ischemic hemi- 

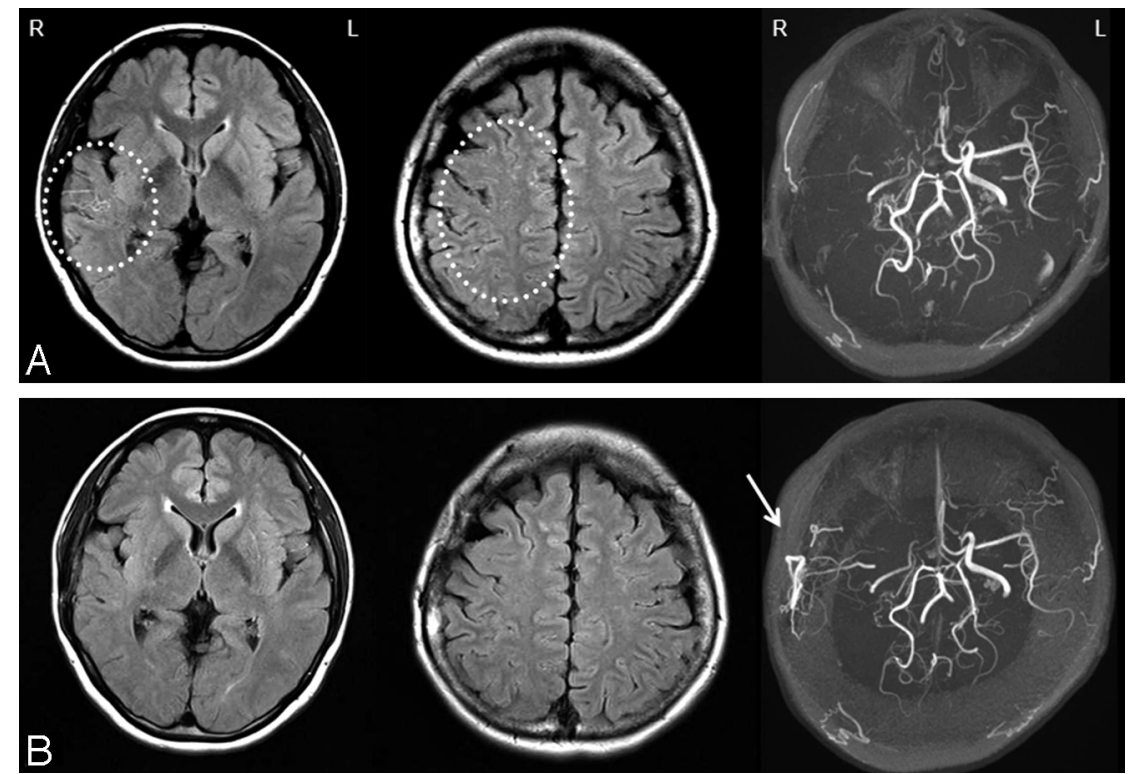

Fig 5. A female patient with unsymmetric ivy sign and decreased ivy sign after operation (10 months follow-up period). She had sustained a left TIA. A, Preoperative FLAIR images showed moderate ivy dominance in the right hemisphere (dotted circles). Preoperative MRA grade in the right and left hemispheres were III and II, respectively. She underwent direct bypass surgery in the right side. B, Postoperative FLAIR image obtained 10 months after revascularization surgery revealed decreased ivy sign in the right hemisphere. Postoperative MRA showed well-developed collateral vessels via bypass in the right MCA region (arrow). The patient had no symptoms after the operation.

sphere where ivy signs were dominant. In addition, all 15 had more misery perfusion areas or showed lower vascular reactivity in the IDH than ILDH, with the consequence that they had cerebral revascularizations on the IDH. On the other hand, 12 patients with symmetric ivy distributions in the hemispheres on MR imaging showed a variety of hemispheric differences in the cerebrovascular reserve. Three of 12 patients underwent bypass surgery because of unilateral and bilateral TIAs. These results demonstrate that the operative side for bypass surgery can be selected from a patient's clinical symptoms and distribution difference of ivy signs on FLAIR images, if available, without performing a quantitative analysis of SPECT with ACT challenge or PET. Intra-extracranial bypass will be established on the ivy-dominant hemisphere causing TIA. An additional investigation with greater numbers of patients is needed to perform more profound analysis between the ivy proliferation and the hemodynamic status. The fact that patients with symmetric ivy distributions showed decreased vascular reserve in either hemisphere should be taken into consideration. For those patients, evaluation of clinical symptoms and quantitative analysis are necessary.

Ohta et $\mathrm{al}^{1}$ first reported that the ivy sign is a diffuse leptomeningeal enhancement found on postcontrast MR images of patients with Moyamoya disease in childhood. The ivy sign is also seen in a child with Moyamoya disease on FLAIR images. ${ }^{2}$ Yoon et $\mathrm{al}^{10}$ reported that postcontrast T1-weighted MR images revealed a leptomeningeal ivy sign in 40 hemispheres (frequency of visualization, 71\% [40/56 hemispheres]), whereas unenhanced FLAIR images depicted the sign in 26 hemispheres (frequency of visualization, 46\% [26/56 hemispheres]). They concluded that contrast-enhanced T1weighted images are better than FLAIR images in the depiction of the leptomeningeal ivy sign in Moyamoya disease. ${ }^{10}$ Because a contrast medium for Moyamoya disease is not routinely used in our institution, the correlation between an ivy sign and contrast enhancement was not investigated. However, the frequency of visualization of ivy signs on FLAIR images in patients with Moyamoya was $97.1 \%$ (34/35 patients) in this study, which is much higher than that reported by Yoon et al. ${ }^{10}$ This might be mainly because MR imaging is performed at 3T, which is twice as high as their level, and partly because of differences in parameter settings. In addition, an MR imaging examination without contrast media is much easier and safer than an MR examination with contrast media.

In our study, we analyzed the correlations between ivy signs and the development of the collateral circulation using MRA and conventional angiography findings. It was found that a more prominent ivy sign was observed in hemispheres with higher MRA stages, where collateral circulation is well developed. This result is consistent with that reported by Fujiwara et al. ${ }^{15}$ The correlation between ivy proliferations and angiographic arteriocapillary circulation time (ACCT) was also studied. There was a tendency for an ivy sign to be prominent in a hemisphere with a prolonged vascular circulation time. From a morphologic standpoint, the ivy sign represents a retrograde slow flow of pial vasculature via the leptomeningeal collaterals shown on an angiographic image. Conversely, patients with Moyamoya disease whose ivy signs were equally observed in both hemispheres tended to show symmetric cerebrovascular reserve and no difference of MRA stage and ACCT between both hemispheres. These findings demonstrate that unilateral hemispheric ivy proliferation represents a development of leptomeningeal collaterals toward the ischemic area where the cerebrovascular reserve is decreased more than at the contralateral side. More than half of ivy sign proliferations had decreased or diminished after successful bypass surgery within a short follow-up period. The ivy sign can play an important role in reflecting the regional hemodynamic status of patients with Moyamoya disease. 


\section{Conclusions}

The ivy sign is seen in hemispheres with diminished cerebrovascular reserve. The unilateral hemispheric ivy proliferation correlates better with a larger misery perfusion area than the contralateral hemisphere associated with the development of leptomeningeal collaterals toward the ischemic area in patients with Moyamoya disease.

\section{References}

1. Ohta T, Tanaka H, Kuroiwa T. Diffuse leptomeningeal enhancement, "ivy sign," in magnetic resonance images of moyamoya disease in childhood: case report. Neurosurgery 1995;37:1009-12

2. Maeda M, Tsuchida C. "Ivy sign" on fluid-attenuated inversion-recovery images in childhood Moyamoya disease. AJNR Am J Neuroradiol 1999;20:1836-38

3. Houkin K, Nakayama N, Kuroda S, et al. Novel magnetic resonance angiography stage grading for Moyamoya disease. Cerebrovasc Dis 2005;20:347-54

4. Fukui M. Guidelines for the diagnosis and treatment of spontaneous occlusion of the circle of Willis ('Moyamoya' disease). Research Committee on Spontaneous Occlusion of the Circle of Willis (Moyamoya Disease) of the Ministry of Health and Welfare, Japan. Clin Neurol Neurosurg 1997;99(Suppl 2):S238-40

5. Yamamoto S, Watanabe M, Uematsu T, et al. Correlation of angiographic circulation time and cerebrovascular reserve by acetazolamide-challenged single photon emission CT. AJNR Am J Neuroradiol 2004;25:242-47

6. Iida $\mathrm{H}$, Itoh $\mathrm{H}, \mathrm{Nakazawa} \mathrm{M}$, et al. Quantitative mapping of regional cerebral blood flow using $\left[{ }^{123} \mathrm{I}\right] \mathrm{N}$-isopropyl- $\rho$-iodoamphetamine (IMP) and single photon emission computed tomography. J Nucl Med 1994;35:2019-30

7. JET Study Group. Japanese EC-IC bypass Trial (JET study): study design and interim analysis. Surg Cereb Stroke 2002;30:97-100

8. Mizumura S, Nakagawara J, Takahashi M, et al. Three-dimensional display in staging hemodynamic brain ischemia for JET study: objective evaluation using SEE analysis and 3D-SSP display. Ann Nucl Med 2004;18:13-21

9. Kawamura S, Ashizaki M, Saida S, et al. Usefulness of rate of increase in SPECT counts in one-day method of N-isopropyl-4-iodoamphetamine [123I] SPECT studies at rest and after acetazolamide challenge using a method for estimating time-dependent distribution at rest. Ann Nucl Med 2008;22:457-63

10. Yoon HK, Shin HJ, Chang YW. "Ivy sign" in childhood Moyamoya disease: depiction on FLAIR and contrast-enhanced T1-weighted MR images. Radiology 2002;223:384-89

11. Ikezaki K, Matsushima T, Kuwabara Y, et al. Cerebral circulation and oxygen metabolism in childhood Moyamoya disease: a perioperative positron emission tomography study. J Neurosurg 1994;81:843-50

12. Nakagawara J, Takeda R, Suematsu K, et al. Quantification of regional cerebral blood flow and vascular reserve in childhood Moyamoya disease using [123I]IMP-ARG method. Clin Neurol Neurosurg 1997;99(Suppl 2):S96-99

13. Nariai T, Suzuki R, Matsushima Y, et al. Surgically induced angiogenesis to compensate for hemodynamic cerebral ischemia. Stroke 1994:25:1014-21

14. Carusone LM, Srinivasan J, Gitelman DR, et al. Hemodynamic response changes in cerebrovascular disease: implications for functional MR imaging. AJNR Am J Neuroradiol 2002;23:1222-28

15. Fujiwara H, Momoshima S, Kuribayashi S. Leptomeningeal high signal intensity (ivy sign) on fluid-attenuated inversion-recovery (FLAIR) MR images in Moyamoya disease. Eur J Radiol 2005;55:224-30 